\title{
Avaliação do conhecimento da população leiga de uma instituição de ensino superior sobre infarto agudo do miocárdio
}

\author{
Evaluation of the knowledge of the lay population of a higher education institution on acute \\ myocardial infarction
}
Evaluación del conocimiento de la población laica de una institución de educación superior sobre el infarto agudo de miocardio

Ramili Ribeiro Correia ${ }^{1 *}$, Eric da Silva ${ }^{1}$, Izolda Santos Matiello ${ }^{1}$, Antônio Tiago da Silva Souza, Antonia Jaírla Oliveira da Silva ${ }^{2}$, Carla Valéria Silva Oliveira ${ }^{1}$, Francisco Ricardo Nascimento Freitas ${ }^{2}$, José Arnaldo de Sá Junior ${ }^{3}$, Marina Carvalho Magalhães Araújoํㅗ Naira Denise de Sousa Santos ${ }^{4}$.

\section{RESUMO}

Objetivo: Identificar o nível de conhecimento da população leiga de uma Instituição de Ensino Superior sobre Infarto Agudo do Miocárdio. Métodos: Estudo do tipo transversal, prospectivo e observacional, realizado em uma Instituição de Ensino Superior do Estado do Piauí. A amostra foi constituída por 340 (trezentos e quarenta) alunos das áreas de humanas e exatas e a análise foi realizada por meio de estatística descritiva. Resultados: $15 \%$ dos entrevistados afirmaram não saber o que é Infarto Agudo do Miocárdio, $64 \%$ não soube o tempo para checar o pulso e identificar uma parada cardíaca e apenas $9 \%$ soube responder qual o local correto para colocar os eletrodos do Desfibrilador Externo Automático. Conclusão: A população estudada apesar de ser caracterizada jovem e com maior nível educacional, falhou em mais da metade das vezes em identificar os fatores de riscos, como os sinais e sintomas, bem como, a conduta indicada nesses casos. $O$ estudo evidenciou que o nível de conhecimento dos entrevistados ainda é considerado insuficiente, revelando a necessidade de intervenção.

Palavras-chave: Infarto do miocárdio, Massagem cardíaca, População.

\begin{abstract}
Objective: To identify the level of knowledge of the lay population of a Higher Education Institution about Acute Myocardial Infarction. Methods: Cross-sectional, prospective and observational study, carried out in a Higher Education Institution in the State of Piauí. The sample consisted of 340 (three hundred and forty) students from the human and exact areas and the analysis was carried out using descriptive statistics. Results: $15 \%$ of respondents said they did not know what Acute Myocardial Infarction is, $64 \%$ did not know the time to check the pulse and identify a cardiac arrest and only $9 \%$ knew how to answer the correct place to place the External Automated Defibrillator electrodes. Conclusion: The studied population, despite being characterized as young and with a higher educational level, failed more than half of the time to identify risk factors, such as signs and symptoms, as well as the conduct indicated in these cases. The study showed that the level of knowledge of the interviewees is still considered insufficient, revealing the need for intervention.
\end{abstract}

Keywords: Myocardial infarction, Heart massage, Population.

\footnotetext{
${ }^{1}$ Centro Universitário de Saúde, Ciências Humanas e Tecnológicas do Piauí (UNINOVAFAPI), Teresina - PI.

*E-mail: ramili05@hotmail.com

2 Universidade Federal do Delta do Parnaíba (UFDPAR), Parnaíba - PI.

${ }^{3}$ Faculdade de Tecnologia de Teresina (CET), Teresina - PI.

${ }^{4}$ Escola de Saúde Pública do Ceará (ESP/CE), Crateús - CE.
} 


\section{RESUMEN}

Objetivo: Identificar el nivel de conocimiento de la población laica de una institución de educación superior sobre el infarto agudo de miocardio. Métodos: Estudio transversal, prospectivo y observacional, realizado en una Institución de Educación Superior del Estado de Piauí. La muestra consistió en 340 (trescientos cuarenta) estudiantes de las áreas humanas y exactas y el análisis se realizó utilizando estadísticas descriptivas. Resultados: El 15\% de los encuestados dijeron que no sabían qué es el infarto agudo de miocardio, el $64 \%$ no sabían el momento de controlar el pulso e identificar un paro cardíaco y solo el $9 \%$ sabían cómo responder al lugar correcto para colocar los electrodos de desfibrilador automático externo. Conclusión: La población estudiada, a pesar de estar caracterizada como joven y con un nivel educativo más alto, no logró identificar más de la mitad de las veces los factores de riesgo, como los signos y síntomas, así como la conducta indicada en estos casos. El estudio mostró que el nivel de conocimiento de los entrevistados todavía se considera insuficiente, lo que revela la necesidad de intervención.

Palabras clave: Infarto del miocardio, Masaje cardíaco, Población.

\section{INTRODUÇÃO}

O infarto agudo do miocárdio (IAM) é a patologia causada pela isquemia prolongada provocando a morte de cardiomiócitos. Tal fato, basicamente, é provocado por duas razões: trombose, sendo a principal causa por trombos, e vasoespamos, sendo menor relacionada e está relacionada com a erosão de placas ateroscleróticas. Os principais fatores de risco relacionados ao IAM é a diabetes, o colesterol elevado, a obsediade, a hipertensão, dentre outros. Entretanto, outro fator é crucial no manejo da doença que é o atendimento pré-hospitalar, pois os cuidados certos na identificação e no procedimento correto do IAM fazem total diferença na sobrevivência do paciente (PESARO AEP, 2004).

Por volta de 1960, iniciou-se um direcionamento mais especializado no atendimento pré-hospitalar nos casos de IAM, devido as altas taxas de mortalidade envolvendo esses casos. As primeiras horas do IAM são vitais, haja vista que $40-65 \%$ das mortes ocorrem na primeira hora do acometimento, além de $80 \%$ dos casos de mortes serem registrados nas primeiras 24 horas. Estes números são na maioria das vezes contemplados em ambientes extra-hospitalares, em sua maioria sem a presença de médicos e por conta de uma parada cardiorrespiratória por fibrilação ventricular (BODANESE LC, et al., 2015).

Uma pesquisa norte-americana mostrou que mais de 12 milhões de pessoas têm alguma doença cardíaca e mais de um milhão apresenta um IAM por ano, resultando em 466.000 mortes relacionadas com a doença arterial coronariana (DAC). (BASTOS AS, et al., 2012). Segundo o Departamento de Informática do Sistema Único de Saúde do Brasil (DATASUS), no ano de 2010, no Brasil, as doenças cardiovasculares foram apontadas como a primeira causa de morte, correspondendo a $29 \%$ dos óbitos. O IAM é a segunda causa de morte mais frequente, $7 \%$ do total de óbitos no referido ano, perdendo para as doenças cardiovasculares em geral (MARCOLINO et al., 2013; BORGES LP, et al., 2019).

Em uma pesquisa brasileira, os pacientes com IAM não procuraram imediatamente os serviços de saúde, pois não reconheceram os sinais e sintomas. As sequelas causadas por pessoas que sofreram de IAM são consideradas crônicas e exigem ações e procedimentos dos serviços de saúde, consequentemente, geram custos mais altos e causam enormes prejuízos para a sociedade, sendo assim, considerado um problema de saúde pública (BASTOS AS, et al, 2012).

As pessoas que sofrem IAM, em sua maioria, apresentam dor precordial em aperto retroesternal, irradiada para membro superior esquerdo, com intensidade elevada e prolongada por mais que 20 minutos, não melhorando ou apenas tendo um alívio estando em repouso ou fazendo uso de nitrato sublingual. Ainda pode irradiar para mandíbula, membro superior direito, dorso, ombros, região epigástrica, apresentando mal-estar, dispneia e taquicardia (PESARO AEP, et al., 2004).

Porém, nem todos os indivíduos reconhecem essas condições clinicas e as ignoram, prolongando assim, o tempo de socorro. Quando comparada aos homens, as mulheres com IAM podem apresentar menor taxa de dor torácica e sudorese, sendo mais frequente a dispneia (MUSSI FC E PEREIRA A, 2010). 
Para o tratamento adequado do IAM há necessidade da interligação de vários setores como as comunidades, unidades de pronto atendimento, serviço de transporte de emergência e hospital com serviços de hemodinâmica e de terapia intensiva (MARCOLINO SM, 2013).

Indivíduos com IAM já instalado deve-se promover recuperação do fluxo sanguíneo coronariano, levando em consideração tempo de socorro. Ou seja, pessoas tratadas na primeira hora de evolução dos sintomas experimentam uma redução significativa da mortalidade hospitalar. Dessa forma, o diagnóstico é de fundamental importância e a agilidade dos indivíduos para reconhecer o infarto e procurar um serviço de saúde tem impacto direto no prognóstico (MUSSI FC, et al., 2014).

Sendo assim, pesquisas que avaliem o conhecimento da população leiga sobre os sinais e sintomas do IAM, além do atendimento inicial em casos que evoluam para Parada Cardiorrespiratória e Cerebral (PCRC), tornam-se imprescindíveis para estabelecer projetos que esclareçam, de forma correta e objetiva, à população leiga e, consequentemente, abrir uma perspectiva de redução da mortalidade pré-hospitalar através de políticas de educação em saúde.

Segundo a American Heart Association - AHA (2015), recomenda-se para socorristas leigos o incentivo ao reconhecimento imediato da ausência de resposta no infartado e que seja feito um programa de educação ao público leigo sobre os procedimentos imediatos, incluindo o uso do desfibrilador para paciente que venha a ter parada cardiorrespiratória extra-hospitalar em locais públicos. Estudos dentro da linha de pesquisa supracitada, mas aplicada ao IAM, não são conhecidos e poderiam impactar no diagnóstico situacional e nível de conhecimento da população leiga, norteando os aspectos que devem ser abordados como parte integrante da educação continuada e que, possivelmente, poderia contribuir para a redução da mortalidade extra hospitalar no infarto.

O presente estudo teve como objetivo identificar o nível de conhecimento de estudantes de uma de uma instituição de ensino superior sobre o IAM. Em seguida, verificar o reconhecimento dos fatores de risco, sinais e sintomas do IAM por essa população. Consequentemente, avaliar o nível de conhecimento dessa população leiga a respeito da conduta indicada para o atendimento inicial do IAM no ambiente extra hospitalar; e sobre a Parada Cardiorrespiratória e Cerebral em vítimas de infarto.

\section{MÉTODOS}

Estudo do tipo transversal, prospectivo e observacional. Realizado em uma Instituição de Ensino Superior (IES) do Estado do Piauí. A coleta dos dados foi realizada no período de julho a agosto de 2017. A população do estudo foi composta por 2259 (dois mil duzentas e cinquenta e nove) alunos das áreas de humanas e exatas, informações essas colhida na secretaria geral dos cursos oferecidos pelo IES. Do total de alunos matriculados na IES nos cursos selecionados para o estudo, optou-se por um cálculo amostral simples de 5\% resultando em 340 (trezentos e quarenta) pessoas entrevistadas. Os critérios de inclusão foram os indivíduos na faixa etária de 18 a 65 anos, leigos em relação ao tema que foi pesquisado. O termo "leigo" definido nesta pesquisa, para evitar vieses, como indivíduos sem formação acadêmica universitária nas áreas de saúde (Enfermagem, Fonoaudiologia, Fisioterapia, Educação Física, Medicina, Nutrição, Odontologia, Biomedicina e Cursos de Primeiros Socorros).

Os critérios de exclusão foram: indivíduos que recusarem a participação no estudo e/ou não conseguirem responder o questionário e ou indivíduos da área de humanas e exatas que já tem ou tiveram formação e conhecimento na área de saúde. A coleta de dados desse estudo teve como ferramenta uma ficha contendo questionários aplicados pelos pesquisadores, contendo a identificação do indivíduo (idade, gênero, etnia, estado civil, nível educacional, renda mensal, profissão) e a segunda parte contendo a avaliação do conhecimento sobre IAM (identificação de fatores de riscos, sinais e sintomas e conduta inicial adequada).

Para a análise estatística do trabalho foi utilizado o software Statistical Package for the Social Science (SPSS) versão 17.0 e BioEstat versão 5.3, além do Microsoft Excel 2013. Para avaliação de normalidade foi utilizado o teste D'agostinho e teste descritivo com definição das frequências absolutas e relativas, medidas de tendência central e medidas de dispersão. Os resultados foram analisados e considerados para significância estatística o valor de $p<0,05$. 
Aos participantes foi apresentado o TCLE e TALE, garantida a proteção da imagem e a privacidade, confidencialidade, a não estigmatização e a não utilização de informações em prejuízo das pessoas, conforme os princípios norteadores dispostos na Resolução no 466/12, do Conselho Nacional de Saúde. Estudo aprovado pelo Comitê de Ética e Pesquisa do UNINOVAFAPI segundo a Resolução 466/12. CAAE: 67150417.7.0000.5210. Todos os aspectos éticos contidos na Resolução 466/2012 que trata de ética envolvendo seres humanos foram obedecidos (BRASIL, 2012).

\section{RESULTADOS}

A amostra foi composta por 340 entrevistados, sendo $55 \%(n=187)$ mulheres e $45 \%(n=153)$ homens. A idade predominante no estudo foi de 18 a 25 anos, correspondendo a 83\% (283) dos indivíduos, os demais se enquadraram na faixa etária de 26 a 50 anos (17\%, $n=57)$. Dentre os entrevistados, $53 \%(n=179)$ declararam não possuírem renda mensal alegando ter unicamente atividade acadêmica. Dentre os cursos das áreas de humanas e exatas oferecidos pela Instituição de Ensino Superior, foi obtido uma maior participação dos alunos dos cursos de Direito 36\% ( $n=122)$, seguido de Arquitetura e Urbanismo 30\% ( $n=103)$ e engenharia civil 19\% ( $n=63)$ (Tabela 1).

Tabela 1 - Caracterização dos estudantes do nível superior entrevistados, n=340. Teresina-PI, 2017.

\begin{tabular}{lll}
\hline Variável & N & $\%$ \\
\hline Sexo & & \\
\hline Masculino & 153 & 45 \\
$\quad$ Feminino & 187 & 55 \\
\hline Idade & 283 & 83 \\
\hline $18-25$ & 57 & 17 \\
$26-50$ & & \\
\hline Renda Mensal & 161 & 47 \\
$\quad$ Possuíam & 179 & 53 \\
$\quad$ Não possuíam & & 36 \\
\hline Cursos & 122 & 30 \\
$\quad$ Direito & 103 & 19 \\
$\quad$ Arquitetura e Urbanismo & 63 & 15 \\
$\quad$ Engenharia Civil & 52 & $\mathbf{1 0 0}$ \\
$\quad$ Outros (4) & $\mathbf{3 4 0}$ & \\
\hline Total & & \\
\hline
\end{tabular}

Fonte: Correia RR, et al., 2017.

Dentre os entrevistados, $15 \%$, afirmaram não saber o que é IAM. Com relação ao perfil demográfico mais acometido pelo IAM, os entrevistados apontaram os homens (33\%) e idosos (39\%) como principal perfil. Uma minoria (18\%) não soube responder. Dentre os fatores de riscos relacionados ao IAM os mais citados foram a hipertensão (29\%) seguido da hipertrigliceridemia (16\%) e alimentação hipercalórica (10\%). 9\% dos entrevistados não souberam responder. Quantos aos sinais e sintomas mais frequentes nos indivíduos durante o IAM, $42 \%$ dos entrevistados apontaram a dor retroesternal, $24 \%$ dormência com fraqueza no braço e mandíbula, e $4 \%$ não souberam responder (Tabela 2).

Quando questionados quanto ao procedimento inicial ao presenciar a suspeita de alguém infartando um pouco mais da metade dos entrevistados (53\%) responderam que ligar para o Serviço de Atendimento Móvel de Urgência (SAMU) seria a conduta. Com relação ao conhecimento do número de contato do SAMU, 62\% acertaram marcando a opção 192, 31\% erraram marcando as opções 190, 191 ou 193 e 7\% não souberam responder. Sobre o tempo mínimo e máximo para checar o pulso e identificar a parada cardíaca após um infarto mais da metade (64\%) dos entrevistados afirmaram não saber. Apenas $9 \%$ dos entrevistados responderam corretamente (Tabela 2). 
Tabela 2 - Conhecimento dos estudantes do ensino superior sobre reconhecimento de IAM, $n=340$. Teresina-PI, 2017.

\begin{tabular}{lll}
\hline Variável & N & $\%$ \\
\hline Significado da sigla IAM & & \\
\hline Conheciam & 289 & 85 \\
Não conheciam & 51 & 15 \\
\hline Identificação do Grupo de Risco & & \\
\hline Homens & 112 & 33 \\
Idosos & 133 & 39 \\
Não Souberam responder & 61 & 18 \\
\hline Identificação de Fatores de risco & & \\
\hline Hipertensão & 99 & 29 \\
Hipertrigliceridemia & 54 & 16 \\
Alimentação hipercalórica & 34 & 10 \\
Outros fatores & 122 & 36 \\
Não Souberam responder & 31 & 9
\end{tabular}

\section{Identificação de Sinais e Sintomas}

\begin{tabular}{lll}
\hline Dor retrosternal & 143 & 42 \\
Dormência com fraqueza no braço e na & 82 & 24 \\
mandíbula & & 30 \\
Outros sinais & 101 & 4 \\
Não souberam responder & 14 & \\
\hline Número do SAMU & & 62 \\
\hline Acertaram (192) & 211 & 31 \\
Erraram (190, 191 ou 193) & 105 & 7 \\
Não souberam responder & 24 & \\
\hline Tempo mínimo e máximo para checar o pulso & & 9 \\
\hline Entre 1-5 segundos & 32 & 4 \\
Entre 3-5 segundos & 13 & 14 \\
Entre 3-10 segundos & 49 & 9 \\
Entre 5-10 segundos & 30 & 64 \\
Não souberam responder & 216 & 100 \\
\hline Total & 340 &
\end{tabular}

Fonte: Correia RR, et al., 2017.

Sobre onde colocar as mãos para comprimir o tórax durante a massagem cardíaca, a maioria (77\%) acertou, ou seja, colocando as mãos sobrepostas e com a região hipotênar sobre o esterno. Os $23 \%$ restantes erraram e responderam uma das outras alternativas. Com relação ao pulso a ser checado para confirmação de parada cardíaca, $47 \%$ responderam adequadamente, ou seja, a artéria carótida, seguida da artéria radial (44\%) (Tabela 3). 
Figura 1 - Possíveis Posicionamentos das Mãos para Massagem Cardíaca Sugerido na Entrevista dos Estudantes da Instituição de Ensino Superior. Teresina, 2017.

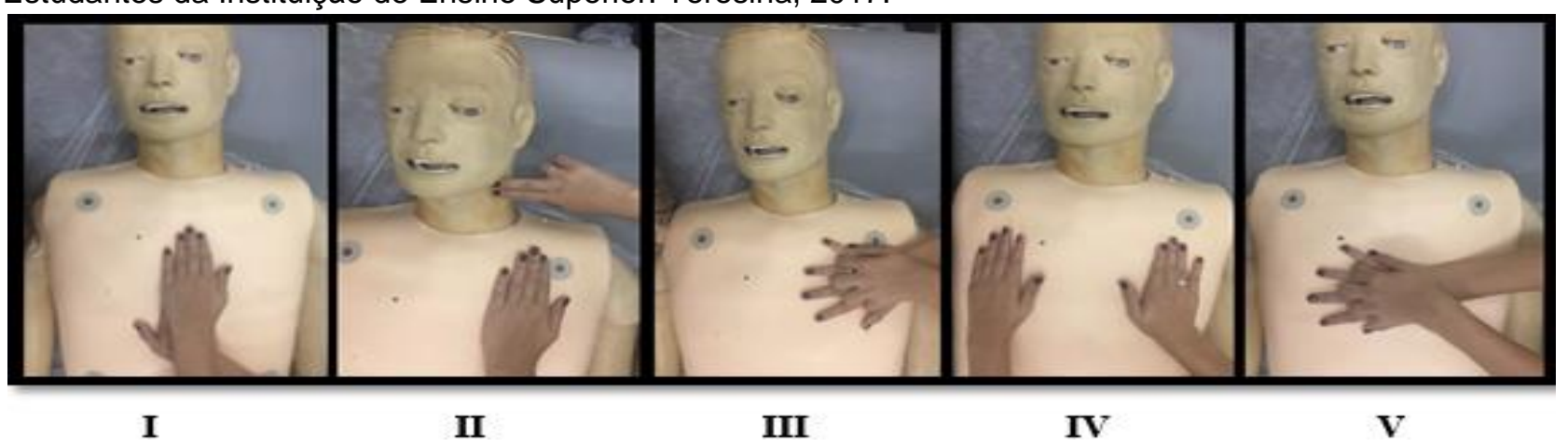

Fonte: Correia RR, et al., 2017

Já ao serem questionados sobre a escolha ideal do pulso para confirmar se o infartado está sofrendo uma parada cardíaca, menos da metade respondeu corretamente: artéria carótida (47\%) (opção IV, da Figura 2). A artéria radial foi selecionada por $44 \%$ dos entrevistados (Tabela 3 ).

Figura 2 - Possíveis Posicionamentos das Mãos para Averiguação da Presença de Pulso Sugerido na Entrevista dos Estudantes da Instituição de Ensino Superior. Teresina, 2017.

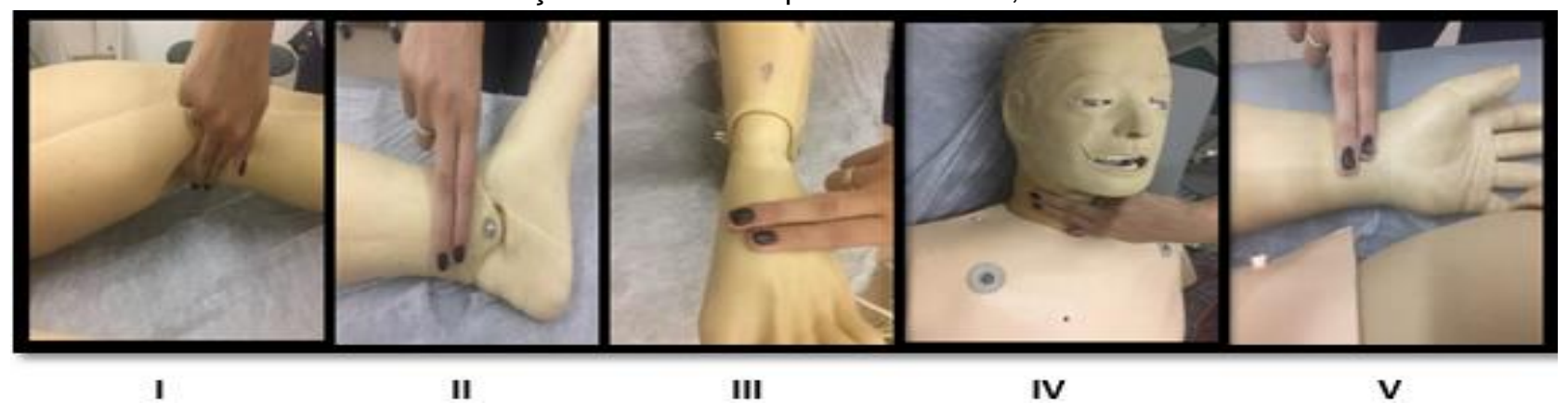

Legenda: Da direita para a esquerda poplíteo (I), tibial (II), pedioso (III), carotídeo (IV) e o radial (V).

Fonte: Correia RR, et al., 2017.

Com relação ao conhecimento sobre a existência do Desfibrilador Elétrico Automático (DEA) para auxiliar a reanimação cardiorrespiratória, $67 \%$ afirmaram que conhecem o dispositivo, e destes, $66 \%$ sabiam a finalidade dele. $14 \%$ dos entrevistados responderam que a sua função é massagear o coração e, $17 \%$ não souberam responder.

Quanto ao local para colocar os eletrodos do DEA caso a pessoa venha a ter uma parada a opção correta somou somente 9\% das escolhas dos entrevistados (Posição V da Figura 3). A opção IV, FIGURA 3 foi selecionada por $69 \%$ dos entrevistados (Tabela 3 ).

Figura 3 - Possíveis Posicionamentos dos Eletrodos do Desfibrilador Externo Automático Sugeridos na Entrevista dos Estudantes da Instituição de Ensino Superior. Teresina, 2017.

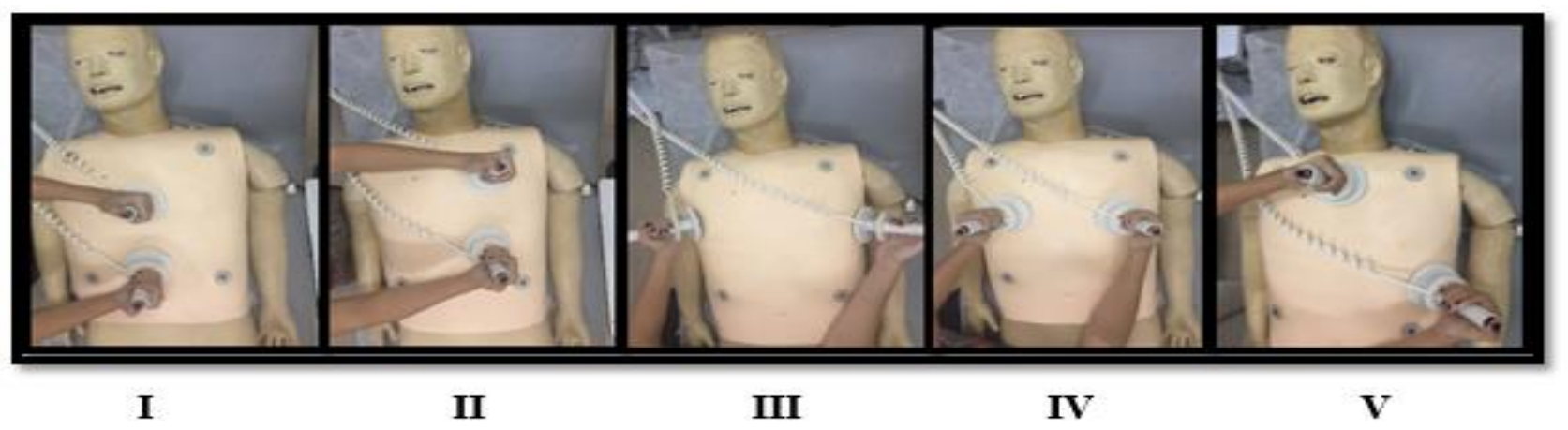

Fonte: Correia RR, et al., 2017. 
Tabela 3 - Conhecimento dos estudantes do ensino superior primeiros socorros, n=340. Teresina-PI, 2017.

\begin{tabular}{llll}
\hline Variável & $\mathbf{N}$ & $\%$ \\
\hline Posicionamento das mãos durante reanimação (Figura 1) & & \\
\hline I & 24 & 7 \\
II & 14 & 4 \\
III & 34 & 10 \\
IV & 10 & 3 \\
V & 258 & 76 \\
\hline
\end{tabular}

Posição das mãos na averiguação do pulso

\begin{tabular}{lll}
\hline I & 7 & 2 \\
II & 3 & 1 \\
III & 20 & 6 \\
IV & 160 & 47 \\
V & 150 & 44
\end{tabular}

\section{Posicionamento dos eletrodos do DEA}

\begin{tabular}{llll}
\hline I & 24 & 7 \\
II & 27 & 8 \\
III & 24 & 7 \\
IV & 234 & 69 \\
V & 31 & 9 \\
\hline Total & & $\mathbf{1 0 0}$ & -
\end{tabular}

Fonte: Correia RR, et al., 2017.

\section{DISCUSSÃO}

Analisando os resultados da amostra entrevistada, foi identificado que $15 \%$ dos entrevistados não sabiam o que é IAM. Este é um dado alarmante, pois as entrevistas foram realizadas com indivíduos cursando nível superior, tendo acima de 18 anos, com livre acesso a fontes de pesquisa e, mesmo assim, declararam não ter conhecimento sobre a principal causa de morte no mundo.

Conforme a Organização Mundial de Saúde (OMS), dentre os 20 milhões de indivíduos que sofrem de doenças cardíacas, aproximadamente 12 milhões são vítimas fatais do Infarto Agudo do Miocárdio (ARAÚJO IFM, et al., 2016).

Avaliando as respostas com relação ao perfil dos indivíduos que sofrem infarto, a maioria dos entrevistados respondeu corretamente que os indivíduos do gênero masculino e acrescentaram que os idosos também fariam parte deste perfil. Sabe-se que a faixa etária mais acometida pelo o IAM são homens acima 40 anos que apresente hábitos de vida sedentário, alimentação hipercalórica, dentre outros, vendo-se que não necessariamente a população idosa está mais sujeita ao IAM. 
Nos últimos anos, as políticas públicas de saúde, têm atentado para o índice de morbimortalidade masculina e eles demonstram a ocorrência de três mortes masculinas para uma feminina. Devido a essa situação catastrófica a saúde do homem passou a ser problema de saúde pública. A principal causa de morte em homens dessa faixa etária 25/59 anos refere-se a ocorrências externas como acidentes, homicídios e outros, seguida das doenças cardiovasculares tendo como a principal o IAM (FONSECA AM, et al., 2013).

Os fatores de risco relacionados ao IAM são as dislipidemias, a hipertensão arterial sistêmica e o diabetes mellitus são consideradas as principais entidades mórbidas com implicações nos maiores índices de morbidade e mortalidade cardiovascular, isso acontece porque os fatores de risco cardiovasculares determinam acelerado processo de envelhecimento dos vasos, fazendo com que mais precocemente ocorra resposta endotelial alterada, acarretando disfunção (LIMA DM, et al., 2018).

O colesterol e triglicerídeos como não se dissolvem totalmente na água, eles são transportados no sangue em forma de partículas chamadas Very Low Density Lipoprotein (VLDL), Low Density Lipoproteins (LDL) e High Density Lipoproteins (HDL) em níveis elevados, aumentam o risco da ocorrência do IAM. Já a nicotina do cigarro provoca um estreitamento da artéria capaz de diminuir a oferta de sangue para as artérias do coração, e, além disso, ela pode aumentar a pressão arterial e o número de batimentos do coração por minuto, aumentando, assim, o risco de IAM (MOURA RI, 2015).

A hipertensão arterial sistêmica foi mais observada, em outros estudos transversais populacionais em áreas urbanas, como um dos principais fatores de risco identificados e justificada esse reconhecimento ineficaz devido à baixa escolaridade (CASCALDI BG, 2014). No presente estudo podemos observar resultados semelhantes com pessoas que estão cursando o ensino de nível superior.

$\mathrm{Na}$ amostra estudada a população reconheceu como sinais e sintomas considerados indicativos do Infarto Agudo do Miocárdio: cefaleia intensa (6\%), dor retroesternal (42\%), alterações na fala (6\%), dor por mais de 20 minutos $(11 \%)$, dor por menos de 20 minutos $(1 \%)$, crise convulsiva $(4 \%)$, parestesia com fraqueza no braço e na mandíbula (24\%), afirmaram não saber (4\%).

Observou-se que os entrevistados conseguiram identificar corretamente os principais sinais e sintomas do IAM. De acordo com a pesquisa feita por Franco B, et al. (2008), 84\% dos pacientes apresentaram dor retroesternal, seguida por dor tipo aperto, sendo que $73,2 \%$ pacientes apresentaram irradiação para outra parte do corpo, como os membros superiores $29,3 \%$.

As atitudes mais frequentes dentre os entrevistados ao ver uma pessoa com suspeita de um IAM foi "chamar o SAMU" (53\%) e, em segundo, seriam as compressões torácicas imediatas (14\%). A maioria dos indivíduos souberam responder corretamente o número do SAMU (62\%) e um número importante de entrevistados (23\%) erraram respondendo 190 mostrando que uma boa parcela da amostra ligaria para o serviço da Polícia Militar.

Estudo realizado por Cruz L et.al. (2017) falava sobre a conscientização e análise de acidentes automobilísticos, dos tipos de sequelas das vítimas e se a população sabia o número do SAMU caso se deparasse com determinada situação. No entanto, ele mostrou que o número mais citado foi 192 e 190 também.

Isso evidencia o desconhecimento da população entrevistada na realização dos procedimentos de primeiros socorros, pois uma parcela relevante não indicou o serviço de emergência como primeira opção e quase $40 \%$ não souberam sequer o número do atendimento. Tal fato, faz com que o procedimento não seja realizado corretamente, haja uma demora muito maior para o socorro das vítimas e. a mortalidade extra hospitalar seja tão elevada em casos como o do IAM.

Sobre o tempo mínimo e máximo para checar o pulso e identificar a parada cardíaca uma grande parcela dos entrevistados respondeu incorretamente. De acordo com estudo de Meireles GOAB (2014), ficou evidente que apenas uma pequena parcela de entrevistados possui preparação para realizar os primeiros socorros, enquanto grande parte tem pouco ou nenhuma preparação. 
Sabe-se que poucas pessoas têm conhecimento de como proceder em casos de emergências e que muitas técnicas são empregadas de forma errônea, agravando ainda mais o estado da vítima. É possível dizer que, infelizmente, o conhecimento sobre primeiros socorros é restrito a determinadas profissões e atividades específicas, sendo, muitas vezes procedimentos pouco utilizados por uma parcela significativa de nossa população.

O DEA tem função vital na sobrevivência e sobrevida dos pacientes sem sequelas e funciona a partir da detecção de arritmias e aplicação de um choque de corrente contínua afim de reverter tal situação. Entretanto, o manuseio do equipamento requere treinamento especializado, sendo de vital importância a adequação ao uso da população leiga afim de amenizar as sequelas do IAM (COSTA MP e MIYADAHIRA AMK, 2008).

Ao serem questionados sobre o que é um DEA, $67 \%$, dos participantes afirmaram saber e, 33\%, não sabiam do que se tratava, dos que responderam sim, $66 \%$, responderam corretamente a função do DEA os demais não souberam responder ou responderam de forma incorreta. Em estudo de Ottoni HF (2015) com soldados foi observado que, 109 soldados $(50,7 \%)$ responderam que conheciam um DEA e sua função e 106 $(49,3 \%)$ disseram que não conheciam o aparelho e suas funções.

Demostrando assim que uma parcela acentuada de pessoas com nível de escolaridade considera boa ainda não sabem que é um DEA e nem suas funções, consequente ente não sabendo usá-lo caso alguém venha a ter uma parada cardiorrespiratória e necessitar de primeiros socorros.

Sobre onde colocar as mãos para comprimir o tórax durante a massagem cardíaca, $77 \%$, dos entrevistados acertam a posição correta. De acordo com estudo feito por Durans CS e Viana JBR (2017) a massagem cardíaca deve ser realizada na altura dos mamilos, dois dedos acima do processo xifóide, com as duas mãos sobrepostas e os cotovelos estendidos. No presente estudo essa questão teve um número de acertos de, $80,9 \%$.

Já segundo artigo de Pergola AM (2009) 8,8\%, dos entrevistados responderam corretamente, 63,4\% parcialmente correto, 18,9\%, de forma incorreta e, 9,4\%, não souberam responder. Demostrando que em ambos os estudos que mais da metade dos entrevistados sabe o devido posicionamento das mãos, porem esse número ainda é insuficiente diante da importância da execução correta da massagem cardíaca. Haja vista, caso seja realizada de forma errada pode ocasionar fraturas nas costelas, contusão pulmonar, fragmentação da cartilagem das costelas e até lacerações no fígado. (DURANS CS e VIANA JBR, 2017)

Na pergunta sobre em qual pulso checar para saber se a pessoa está sofrendo uma parada cardíaca, a artéria a ser checada segundo Aguiar JBN e Andrade EGS (2018) é a carótida (no pescoço) para certificarse da ausência de batimentos cardíacos, devido ser a artéria que mais reflete o arco aórtico. Menos da metade dos indivíduos que participaram da pesquisa acertou a resposta, mostrando uma falta de conhecimento acentuada e uma possível leitura alterada dos batimentos cardíacos.

Ao serem questionados quanto ao uso correto dos eletrodos do DEA, apenas $6 \%$ da amostra respondeu corretamente, mostrando o total desconhecimento da população, resultado equivalente foi observado em estudo de Boaventura AP (2011), que mostrou que nenhum dos $173(100 \%)$ alunos executou corretamente as manobras referente ao DEA. Já é comprovado que o uso correto do DEA pode salvar vidas, mas pode-se observar que só uma pequena parte da população saberia usá-lo em caso de emergência.

\section{CONCLUSÃO}

O estudo evidenciou que o nível de conhecimento da população leiga sobre infarto agudo do miocárdio em uma Instituição de ensino superior do Estado do Piauí ainda é considerado insuficiente. A população estudada apesar de se caracterizar por uma população jovem e com maior nível educacional, falhou em mais da metade das vezes em identificar os fatores de riscos, como os sinais e sintomas, bem como, a conduta indicada nesses casos. Deste modo, o estudo espera ter contribuído para a comunidade científica apontando as lacunas de conhecimento que a população alvo demonstrou incitando possíveis trabalhos ou intervenções futuras no manejo dos sinais e sintomas, fatores de risco, assim como na assistência inicial pré-hospitalar a fim de possibilitar um atendimento mais rápido e assertivo de vítimas do infarto agudo do miocárdio. 


\section{REFERÊNCIAS}

1. AGUIAR JBN, ANDRADE EGS. Conhecimento da equipe de enfermagem sobre o protocolo de ressuscitação cardiorrespiratória no setor de emergência. Rev Inic Cient Ext, 2018; 1(Esp 4):334-341.

2. ARAÚJO IFM, et al. Perfil da população acometida por infarto agudo do miocárdio. Revista enfermagem UFPE online, 2016; 10(7): 2302-2309.

3. AMERICAN HEART ASSOCIATION (AHA). Atualizações das diretrizes RCP e ACE, 2015. Disponível em: https://eccguidelines. heart.org/wp-content/uploads/2015/10/2015-AHA-Guidelines-Highlights-Portuguese.pdf.

4. BASTOS AS, et al. Tempo de chegada do paciente com infarto agudo do miocárdio em unidade de emergência. Revista Brasileira Cir. Cardiovascular, 2012; 27(3): 411-418.

5. BOAVENTURA AP. Avaliação do processo ensino aprendizagem das manobras de ressuscitação cardiorrespiratória (RCP) utilizando o desfibrilador externo automático (DEA): alunos de graduação da área da saúde. Tese (Doutorado em Enfermagem) - Escola de Enfermagem. Universidade de São Paulo, São Paulo, 2011; 143 p.

6. BODANESE LC, et al. Diretrizes da Sociedade Brasileira de Cardiologia Pocket Book 2013-2015. Rio de Janeiro: SBC, 2015; $603 \mathrm{p}$.

7. BORGES LP, et al. Utilização de biomarcadores cardíacos na detecção de infarto agudo do miocárdio. Revista Eletrônica Acervo Saúde, 2019; 11(13): e940.

8. CASCALDI BG, et al. Infarto Agudo do Miocárdio sob a Ótica da População Brasileira. Rev Bras Cardiol. 2014;27(6):409-417

9. CRUZ MP, et al. Abordagem extensionista na Semana Nacional do Trânsito 2015: conscientização e análise de acidentes, de sequelas e do número do SAMU. Revista Brasileira de Extensão Universitária, 2017; 8(1): 9-13.

10. COSTA MP, MIYADAHIRA AMK. Desfibriladores externos automáticos (DEA) no atendimento pré-hospitalar e acesso público à desfibrilação: uma necessidade real. O Mundo da Saúde São Paulo, 2008; 32(1):8-15.

11. DURANS CS, VIANA JBR. Nível de conhecimento em primeiros socorros de professores de educação física, na cidade de Ji-Paraná. Rev. Acta Brasileira do Movimento Humano, 2017; 6(3): 40-55.

12. FONSECA AM, et.al. Infarto agudo do miocárdio: Levantamento de sua ocorrência em homens atendidos de 2008 2012 em um serviço de urgência e emergência de Passos (MG). Revista Ciência et Práxis, 2013; 6(12):29-34.

13. FRACO $B$, et al. Pacientes com infarto agudo do miocárdio e os fatores que interferem na procura por serviço de emergência: implicações para a educação em saúde. Revista Latino-em Enfermagem. 2008; 16(3): 414-418.

14. LIMA DM, et al. Fatores preditores para Infarto Agudo do Miocárdio (IAM) em adultos jovens. Rev. Ciências Biológicas e de Saúde Unit, 2018; 5(1): 203-216.

15. MARCOLINO SM, et al. Implantação da Linha de Cuidado do Infarto Agudo do Miocárdio no Município de Belo Horizonte. Revista Arq. Bras. Cardiol, 2013; 100(4): 307-314.

16. MOURA RI. Relações socioambientais: os casos de mortalidade por infarto do miocárdio na cidade de Teresina-PI. Tese (Doutorado em Geografia) - Instituto de Geociências e Ciências Exatas. Universidade Estadual Paulista, Rio Claro, 2015; $171 \mathrm{p.}$.

17. MUSSI FC, et al. Fatores ambientais associados ao tempo de decisão para procura de atendimento no infarto do miocárdio. Revista Bras. Enfermagem. 2014; 67(5): 722-729

18. MUSSI FC, PEREIRA A. Tolerância a dor no infarto do miocárdio. Revista Acta Paul Enfermagem, 2010; 23(1): 8087.

19. MEIRELES GOAB. A Abordagem de Primeiros Socorros Realizada Pelos Professores em uma Unidade de Ensino Estadual em Anápolis - GO. Rev. Ensaios Cienc., Cienc. Biol. Agrar. Saúde, 2014; 18(1): 25-30.

20. OTTONI HF. Avaliação de ensino e aprendizagem sobre Suporte Básico de Vida (BLS) em recrutas da Força Aérea Brasileira. Revista Em Extensão, Uberlândia. 2015; 14(1): 76-90.

21. PERGOLA AM, ARAUJO IEM. O leigo e o suporte básico de vida. Rev Esc Enferm USP. 2009; 43(2):335-342.

22. PESARO AEP, et al. Infarto agudo do miocárdio - síndrome coronariana aguda com supra desnível supra desnível do segmento st. Revista Assoc. Med. Brasileira. 2004; 50(2): 214-220. 